\title{
Molecular and Multiscale Simulation of Liquid-Vapor Phase Change Heat Transfer
}

\author{
Yuwen Zhang \\ James C. Dowell Professor and Chairman \\ Department of Mechanical and Aerospace Engineering \\ University of Missouri, Columbia, MO 65211, USA \\ zhangyu@missouri.edu
}

Molecular dynamics is a computer simulation technique that the time evolution of a set of interacting molecules is followed deterministically by solving the equations of motion of classical multi-body systems following Newton's second law. Rapid boiling of liquid water film heated by a hot copper plate as well boiling of liquid argon on a nanostructured surface are simulated using molecular dynamics. The results show that liquid molecules close to the plate are instantly overheated and undergo a rapid phase change process. The nanostructure has significant effect on evaporation /boiling of thin film. The degrees of superheat and size of nanoparticles have significant effects on the trajectories of particles and net evaporation rate. Effects of nanostructured defects of copper solid surface on the bubble growth in liquid argon have been investigated through a hybrid atomistic-continuum method. Phase change of argon on five nanostructures has been observed and analyzed. The results showed that the solid surface with wedge defect tends to induce a nano-bubble relatively more easily than the others, and the larger the size of the defect is the easier the bubble generate. 